\title{
Tunable and noncytotoxic PET/SPECT-MRI multimodality imaging probes using colloidally stable ligand-free superparamagnetic iron oxide nanoparticles
}

\author{
This article was published in the following Dove Press journal: \\ International Journal of Nanomedicine \\ 27 January 2017 \\ Number of times this article has been viewed
}

\author{
TH Nguyen Pham' \\ Nigel A Lengkeek ${ }^{2}$ \\ Ivan Greguric ${ }^{2}$ \\ Byung J Kim' \\ Paul A Pellegrini ${ }^{2}$ \\ Stephanie A Bickley ${ }^{3}$ \\ Marcel R Tanudji ${ }^{3}$ \\ Stephen K Jones ${ }^{3}$ \\ Brian S Hawkett' \\ Binh TT Pham'
}

'Key Centre for Polymers and Colloids, School of Chemistry, University of Sydney, ${ }^{2}$ Radioisotopes and Radiotracers, NSTLI, Australian Nuclear Science and Technology Organisation, Sydney, ${ }^{3}$ Sirtex Medical Limited, North Sydney, NSW, Australia

Correspondence: Brian S Hawkett; Binh TT Pham

School of Chemistry, The University of Sydney, Building FI I, Eastern Avenue, Sydney, NSW 2006, Australia

Tel +6I 2 935I 6973; +6I 2 935I 6704

Fax +6I 2 935I 865।

Email brian.hawkett@sydney.edu.au; binh.pham@sydney.edu.au

\begin{abstract}
Physiologically stable multimodality imaging probes for positron emission tomography/single-photon emission computed tomography (PET/SPECT)-magnetic resonance imaging (MRI) were synthesized using the superparamagnetic maghemite iron oxide $\left(\gamma-\mathrm{Fe}_{2} \mathrm{O}_{3}\right)$ nanoparticles (SPIONs). The SPIONs were sterically stabilized with a finely tuned mixture of diblock copolymers with either methoxypolyethylene glycol (MPEG) or primary amine $\mathrm{NH}_{2}$ end groups. The radioisotope for PET or SPECT imaging was incorporated with the SPIONs at high temperature. ${ }^{57} \mathrm{Co}^{2+}$ ions with a long half-life of 270.9 days were used as a model for the radiotracer to study the kinetics of radiolabeling, characterization, and the stability of the radiolabeled SPIONs. Radioactive ${ }^{67} \mathrm{Ga}^{3+}$ and $\mathrm{Cu}^{2+}$-labeled SPIONs were also produced successfully using the optimized conditions from the ${ }^{57} \mathrm{Co}^{2+}$-labeling process. No free radioisotopes were detected in the aqueous phase for the radiolabeled SPIONs 1 week after dispersion in phosphate-buffered saline (PBS). All labeled SPIONs were not only well dispersed and stable under physiological conditions but also noncytotoxic in vitro. The ability to design and produce physiologically stable radiolabeled magnetic nanoparticles with a finely controlled number of functionalizable end groups on the SPIONs enables the generation of a desirable and biologically compatible multimodality PET/SPECT-MRI agent on a single T2 contrast MRI probe.
\end{abstract}

Keywords: magnetic resonance imaging, positron emission tomography, single-photon emission computed tomography, SPIONs, radiolabeling

\section{Introduction}

Over the last two decades, nanotechnology has been extensively developed and applied in the field of imaging technology. Compared to the conventional use of single molecules as contrast agents for in vivo and in vitro imaging, nanoparticles and nanoparticulate assemblies offer significant advantages and are providing important contributions to the field of diagnostic and noninvasive molecular imaging. Due to their high surface areato-volume ratio, a higher amount of the desired payload can be incorporated with the contrast-generating nanoparticles/nanoparticulates, resulting in great improvements in the detection capability. Nanoparticles may allow imaging technologies to achieve a higher level of both anatomical and biological information with the use of a single probe.

The most popular dual-modality imaging technique in research involves a combination of UV/visible absorbance and/or fluorescence with magnetic resonance imaging (MRI). Nanoparticles with inherent optical properties, such as gold and quantum dots, have been labeled with a fluorophore or with an MRI contrast agent, 
allowing their position to be pinpointed via MRI, while providing information on their environment via optical fluorescent signals. ${ }^{1-7}$ Despite the disadvantages of using a negative contrast agent (T2 weighted) in MRI, due to the low signal-to-noise ratio, significant advances over the last 25 years have made the use of magnetic nanoparticles as $\mathrm{T} 2$ contrast agents preferable to the use of nanoparticles as T1-weighted contrast agents. Iron oxide magnetic nanoparticles, due to their inherent magnetic properties and feasibility to produce iron oxide cores, are the most common negative (T2 weighted) contrast agents and have been widely applied to in vitro and in vivo studies. ${ }^{8-12}$ Among the dual-modality approaches, positron emission tomography/single-photon emission computed tomography (PET/SPECT)-MRI technologies offer high spatial resolution $(\sim 50 \mu \mathrm{m}),{ }^{13}$ the best soft tissue contrast, the highest sensitivity, and detection limit. ${ }^{14}$ The most common radionuclide probes applied as radiotracers or therapeutic agents in preclinical and clinical practice are ${ }^{18} \mathrm{~F},{ }^{64} \mathrm{Cu},{ }^{67} \mathrm{Ga},{ }^{68} \mathrm{Ga},{ }^{89} \mathrm{Zr},{ }^{90} \mathrm{Y},{ }^{124} \mathrm{I},{ }^{99 \mathrm{~m}} \mathrm{Tc},{ }^{111} \mathrm{In}$, and ${ }^{177} \mathrm{Lu} .{ }^{12,15-18}$ These radionuclides have been incorporated into nanoparticles via coordination bonds with suitable functional groups or chelating ligands on the nanoparticles.

Despite the fact that hybrid PET/SPECT-MRI technologies have been emerging as new tools utilizing molecular probes for in vivo biomolecular and cellular imaging, as well as (pre-) clinical imaging in medical theranostics (a combination of therapy and diagnostics), a number of challenges in the production and performance of suitable and safe multimodality probes remain. The first challenge is the manufacture of imaging probes for clinical application. Robust synthesis, uniformity, batch-to-batch reproducibility, scaling-up, and colloidal stability remain aspects to be developed and optimized. ${ }^{19}$ The second and most concerning challenge is probe biosafety, in particular their side effects when used in vivo and in clinical applications. Recently, systemic fibrosis was detected in clinical patients who had previously undergone administration of imaging contrast agents for MRI scans. ${ }^{9,20,21}$ This side effect is suggested to be associated with the loss of toxic metal ions from the contrast agents. ${ }^{20}$ In March 2015, the US Food and Drug Administration issued a report warning about the application of Feraheme ${ }^{\mathrm{TM}}$ (AMAG Pharmaceuticals, Lexington, MA, USA), an iron oxide-based product, in the treatment of anemia due to a number of reported serious side effects in patients. ${ }^{22}$ These challenges are likely the reason why nanoparticle platforms remain limited to preclinical use, despite their potential applications in theranostics. ${ }^{23}$

Our previous studies showed that the sterically stabilized superparamagnetic iron oxide nanoparticles (s-SPIONs) were stable under physiological conditions, noncytotoxic in vitro, and have shown potential biomedical applications in drug delivery and stem cell tracking. ${ }^{11,24}$ Our recent publication further demonstrated that these sterically stabilized SPIONs were highly stable and well dispersed after being mixed with fresh human red blood cells. ${ }^{25}$ This work presents a robust aqueous-based production of multimodality PET/SPECTMRI probes on single core superparamagnetic iron oxide (maghemite $\gamma-\mathrm{Fe}_{2} \mathrm{O}_{3}$ ) nanoparticles (SPIONs). Two types of sterically stabilized SPIONs are used in this study: $10 \mathrm{~nm}$ diameter (small core) and $25 \mathrm{~nm}$ diameter (big core) SPIONs with T2 relaxivity values of $368 \mathrm{~s}^{-1} \mathrm{mM}^{-1}$ and $953 \mathrm{~s}^{-1} \mathrm{mM}^{-1}$, respectively. ${ }^{10,11}$ This potentially offers better negative MRI contrast agents than all commercially and clinically available samples of similar core sizes. Ligand-free radiolabeling, which was developed recently for commercially available iron oxide nanoparticles with PET radiotracers, ${ }^{26,27}$ is applied to incorporate metal ions of $\mathrm{Co}, \mathrm{Ga}$, and $\mathrm{Cu}$ as models for PET/SPECT radiotracers. Radiolabeling kinetics, stability, and cytotoxicity of the radiolabeled SPIONs are also investigated.

\section{Materials and methods Materials}

Two different core sizes of the maghemite iron oxide $\left(\gamma-\mathrm{Fe}_{2} \mathrm{O}_{3}\right)$ with average diameter of $10 \mathrm{~nm}$ (SPION10) and $25 \mathrm{~nm}$ (SPION25) were used in this study. The SPIONs were sterically stabilized with a mixture of $5 \% \mathrm{NH}_{2}$ and 95\% methoxypolyethylene glycol (MPEG, molar ratio) end functionalized macro-reversible addition fragmentation chain transfer (RAFT) blocks as reported in our previous publication ${ }^{11,24}$ and referred to herein as s-SPION10 and s-SPION25. All the SPION samples, both uncoated and coated particles, were diluted with Milli-Q water to a concentration of $1 \mathrm{wt} \%$ prior to radiolabeling.

Cobalt(II) nitrate hexahydrate $\left(\mathrm{Co}\left(\mathrm{NO}_{3}\right)_{2} \cdot 6 \mathrm{H}_{2} \mathrm{O}\right.$, $99.999 \%$ trace metals basis), gallium(III) chloride $\left(\mathrm{GaCl}_{3}\right.$, 99.999\% trace metals basis), copper(II) nitrate trihydrate $\left(\mathrm{Cu}\left(\mathrm{NO}_{3}\right)_{2} \cdot 3 \mathrm{H}_{2} \mathrm{O},>99 \%\right)$, and hydrochloric acid $(\mathrm{HCl}$, $30 \%-35 \%$, TraceSELECT ${ }^{\circledR}$ Ultra) were purchased from Sigma-Aldrich (St. Louis, MO, USA). Milli-Q water was used throughout this work. Cell culture media were purchased from Thermo Fisher Scientific (Waltham, MA, USA). All cell lines used in the in vitro tests were from ATCC (Manassas, VA, USA). Cobalt-57 chloride $\left({ }^{57} \mathrm{CoCl}_{2}\right)$ in $0.02 \mathrm{M}$ hydrochloric acid was purchased from Perkin Elmer (Waltham, MA, USA). Gallium-67 chloride $\left({ }^{67} \mathrm{GaCl}_{3}\right)$ in $0.1 \mathrm{M}$ hydrochloric acid suitable for radiochemistry was produced from gallium-67 citrate for injection (Lantheus Medical Imaging, Billerica, MA, USA) using established 
procedures. ${ }^{28}$ Copper-64 chloride $\left({ }^{64} \mathrm{CuCl}_{2}\right)$ in $0.02 \mathrm{M} \mathrm{HCl}$ was obtained from RAPID Labs, SCGH (Perth, Australia).

\section{Experimental methods}

Labeling the sterically stabilized SPIONs with $\mathrm{Co}^{2+}$, $\mathrm{Ga}^{3+}$, and $\mathrm{Cu}^{2+}$

Scheme 1 shows the chemical structures and schematic pictures of the stabilized SPIONs. All of the SPION samples, both uncoated and coated particles, were diluted with Milli-Q water to a concentration of $1 \mathrm{wt} \%$ prior to labeling.

Reactions of SPIONs ( $1 \mathrm{wt} \%, 50 \mu \mathrm{L})$ with Co(II) nitrate, $\mathrm{Ga}$ (III) chloride, or $\mathrm{Cu}$ (II) nitrate ( $1 \mathrm{mM}$ in $0.1 \mathrm{M} \mathrm{HCl}, 40 \mu \mathrm{L}$ ) were carried out at different $\mathrm{pH}$ values and temperatures. Sodium acetate buffers ( $\mathrm{pH} 4,4.5,5,5.5$, and $1.0 \mathrm{M})$ and sodium acetate $(1.0 \mathrm{M})$ were added to the reaction mixtures to the final concentrations of $0.1 \mathrm{M}$. The reaction mixtures $(400 \mu \mathrm{L})$ were then heated at either $75^{\circ} \mathrm{C}$ or $95^{\circ} \mathrm{C}$ using an oil bath for $30 \mathrm{~min}$, after which the free metal ions were separated from the SPIONs by centrifuging at 10,000 rpm for $30 \mathrm{~min}$. After removing supernatant, the SPIONs were washed twice with Milli-Q water and then redispersed in $400 \mu \mathrm{L}$ Milli-Q water. Metal concentrations in the reaction mixtures, supernatants, and products were analyzed by graphite furnace atomic absorption spectroscopy (GF-AAS).

\section{Radiolabeling of SPIONs with ${ }^{57} \mathrm{Co}$ and ${ }^{67} \mathrm{Ga}$ Preparation of $\left[{ }^{57} \mathrm{Co} / \text { natural (nat) } \mathrm{Co}\right]^{2+}$ radiotracer stock solution}

Serial solutions of $\mathrm{Co}\left(\mathrm{NO}_{3}\right)_{2}(0.10,1.0$, and $10 \mathrm{mM}$ in $0.1 \mathrm{M}$ $\mathrm{HCl})$ were prepared by diluting $0.1 \mathrm{M} \mathrm{Co}\left(\mathrm{NO}_{3}\right)_{2}$ stock $(2.91 \mathrm{~g}$
$\mathrm{Co}\left(\mathrm{NO}_{3}\right)_{2} \cdot 6 \mathrm{H}_{2} \mathrm{O}$ in $10 \mathrm{~mL}$ of $\left.0.1 \mathrm{M} \mathrm{HCl}\right)$ in $0.1 \mathrm{M} \mathrm{HCl}$. An aliquot of ${ }^{57} \mathrm{Co}$ in $0.1 \mathrm{M} \mathrm{HCl}(\sim 7 \mathrm{MBq})$ was mixed with the stock solutions of $\mathrm{Co}\left(\mathrm{NO}_{3}\right)_{2}(0.1$ or $1 \mathrm{mM})$ to give a final activity of $\sim 300 \mathrm{kBq}$ per reaction prior to the radiolabeling.

\section{Radiolabeling of SPIONs}

Each radiolabeling experimental condition was carried out in triplicate to establish the reproducibility of the applied process.

Radiolabeling of SPIONs with $\left.\left[{ }^{57} \mathrm{Co} /{ }^{\text {/nat }} \mathrm{Co}\right]\right]^{2+}(\sim 1 \mathrm{kBq} / \mathrm{nmol})$ was carried out in sodium acetate buffer (final concentration of $0.1 \mathrm{M}$ ). Typically, an aliquot of $1.0 \mathrm{M}$ sodium acetate $(40 \mu \mathrm{L})$ was added into an acid-washed $1.5 \mathrm{~mL}$ microcentrifuge tube, followed by the addition of Milli-Q water $\left.(300 \mu \mathrm{L}),\left[{ }^{57} \mathrm{Co} /{ }^{\text {nat }} \mathrm{Co}\right]\right]^{2+}$ tracer solution, and $1 \mathrm{wt} \%$ SPION suspension $(50 \mu \mathrm{L}, 0.5 \mathrm{mg}$ of $\mathrm{Fe})$. The reaction mixture was placed on a heating block at a targeted temperature for $30 \mathrm{~min}$. After cooling for $10 \mathrm{~min}, 20 \mu \mathrm{L}$ of reaction mixture was aliquoted from the reaction mixture, and the radioactivity was measured using a gamma counter. The remaining portion was centrifuged at $10,000 \times g$ for $20 \mathrm{~min}$. Then, $20 \mu \mathrm{L}$ of the supernatant, containing unreacted $\left[{ }^{57} \mathrm{Co} /{ }^{\text {/nat }} \mathrm{Co}\right]^{2+}$, was aspirated out to measure the radioactivity; the remaining supernatant was discarded. The radiolabeled SPIONs were redispersed in Milli-Q water using a sonic bath for 10 min. The control samples were prepared, in which the volume of SPIONs was replaced by Milli-Q water. The radiolabeling efficiencies were calculated as the percentages of (total radioactivity - radioactivity of supernatant)/total radioactivity.
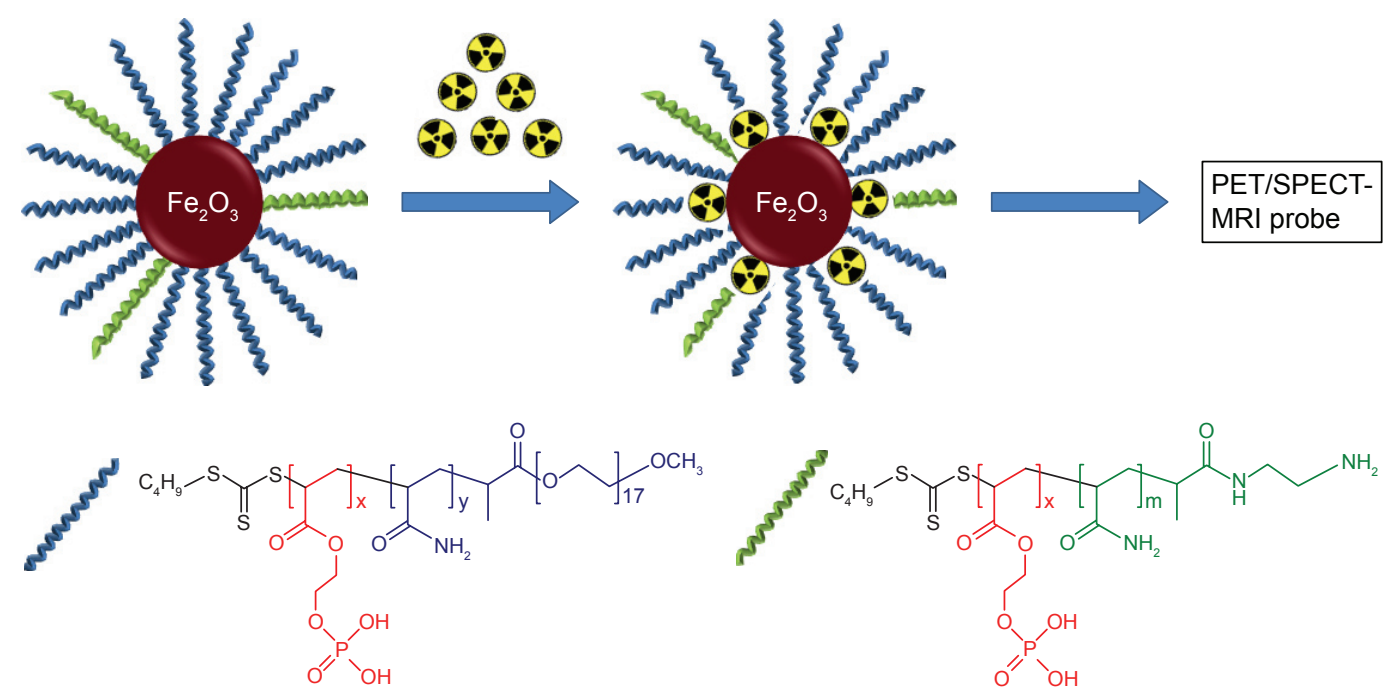

Scheme I Schematic composition of the sterically stabilized SPIONs and the radiolabeled products.

Notes: The steric stabilizers for SPIONs contained anchoring phosphate blocks (red) to iron oxide core surface and stabilizing blocks of $95 \%$ (by molar ratio) MPEG (blue) and $5 \% \mathrm{NH}_{2}$ end functionalized polyacrylamide (green) ( $\mathrm{x}=6 ; \mathrm{y}=0$ and 40 for small and big cores, respectively; $\mathrm{m}=20$ and 60 for small and big cores, respectively).

Abbreviations: SPIONs, superparamagnetic iron oxide nanoparticles; MPEG, methoxypolyethylene glycol; PET, positron emission tomography; SPECT, single-photon emission computed tomography; MRI, magnetic resonance imaging. 
A similar procedure was also applied for the labeling of SPIONs with ${ }^{67} \mathrm{Ga}^{3+}$ and (nonradioactive) $\mathrm{Cu}^{2+}$ unless stated otherwise.

\section{Stability studies in physiological media}

Radiolabeled SPIONs were redispersed in different media, including $0.9 \%$ saline, phosphate-buffered saline (PBS), or $5 \%$ glucose solution, by sonication for $10 \mathrm{~min}$ to check their dispersity. The dispersions were maintained at room temperature for up to 7 days to check their stability. At various time intervals, the dispersions were centrifuged at $10,000 \mathrm{rpm}$ for $20 \mathrm{~min}$. Then, $20 \mu \mathrm{L}$ of the dispersion (before centrifugation) and of the supernatant (after centrifugation) were collected to measure radioactivity on the gamma counter. The particles pellet was redispersed in the media via sonication.

\section{Characterization methods}

\section{Dynamic light scattering (DLS) and transmission electron microscopy (TEM)}

A DLS instrument (Zetasizer nanoseries, helium-neon laser at $633 \mathrm{~nm}, 40 \mathrm{~mW}$; Malvern Instruments, Malvern, UK) with a detection angle of $173^{\circ}$ was used to measure the particle sizes. Samples were measured at $25^{\circ} \mathrm{C}$. The particles were dispersed in Milli-Q water or PBS at $0.5 \mathrm{mg} / \mathrm{mL}$ prior to measurement.

TEM analysis was performed in a JEOL 1400 at an accelerating voltage of $200 \mathrm{kV}$. A diluted sample (about $10 \mu \mathrm{g} / \mathrm{mL}$ in water) of SPIONs was drop casted onto a 200 meshed carbon-coated Formvar ${ }^{\mathrm{TM}}$ copper TEM grid and air-dried before imaging.

\section{Atomic absorption spectroscopy (AAS) to measure metal contents in SPIONs}

The iron content in the SPIONs was measured using an AAS instrument (Varian AA800 spectrometer, with acetylene/air flame atomization). An iron standard solution $(1,000 \mathrm{mg} / \mathrm{L}$ in $\mathrm{HNO}_{3}$; Merck, Darmstadt, Germany) was used for the calibration curve. Samples of dried SPIONs were digested in nitric acid (trace analysis grade, EM-1.00441.1000, 65\% [w/v]; Millipore, Billerica, MA, USA), followed by a dilution with $0.1 \mathrm{M} \mathrm{HCl}$ (Merck) prior to the measurement.

Concentrations of cobalt, gallium, and copper were analyzed using a GF-AAS (Agilent 240FS AA spectrometer with a Zeeman graphite tube atomizer). The standard solutions of $\mathrm{Co}$ (II), $\mathrm{Ga}(\mathrm{III})$, and $\mathrm{Cu}(\mathrm{II})$ at 25,50 , and $40 \mathrm{ppb}$, respectively, in $0.1 \mathrm{M}$ $\mathrm{HCl}$ were used for the calibration curve of each element.

\section{Gamma Counter to measure radioactivity of the radiolabeled SPIONs}

The radiolabeling efficiency for SPIONs labeled with ${ }^{57} \mathrm{Co}$ or ${ }^{67} \mathrm{Ga}$, under different conditions, was determined by gamma counting of the particles and supernatant solutions using a Gamma Counter (Wizard 2480; Perkin Elmer). The particles suspension in an Eppendorf tube was centrifuged at $14,000 \times \mathrm{g}$ for $20 \mathrm{~min}$ to obtain clear supernatant. Then, $10 \mu \mathrm{L}$ of the particle suspension or the supernatant from each SPION sample, in triplicate, was placed in a glass culture tube $(6 \times 50 \mathrm{~mm})$, and the radioactivity was measured on a gamma counter ( $300 \mathrm{~s}$ count time for each measurement).

\section{Cell culture and viability assay}

3T3-L1 and L6 cells (kind gifts from Dr Aviva Levina) were maintained as confluent monolayers in Dulbecco's Modified Eagle's Medium (DMEM, 10566-016; Gibco, Grand Island, NY, USA), supplemented with $10 \%(\mathrm{v} / \mathrm{v})$ fetal calf serum (FCS). The cells were incubated under standard culturing conditions (at $37^{\circ} \mathrm{C}$ with $5 \%$ [v/v] $\mathrm{CO}_{2}$ under humidified conditions). Cells were routinely subcultured by removal of the medium, washing with PBS (IVT3001302; In Vitro Technologies, Noble Park North, VIC, Australia), and detached from the culture-ware by $0.25 \%(\mathrm{w} / \mathrm{v})$ trypsin $(59430 \mathrm{C}$; SAFC Biosciences, Brooklyn, VIC, Australia). The cell suspension was collected in a centrifuge tube with the addition of complete growth medium and spun down at $\sim 1,000 \mathrm{rpm}$ for $3 \mathrm{~min}$. The supernatant was discarded, and the cells were resuspended in fresh growth medium. Cells were then counted on a hemocytometer, and an appropriate number of cells were seeded for further experiments and subculturing.

A total of $1 \times 10^{5}$ cells/well suspension was seeded into each well of a 96-well plate (3599; Corning, Kennebunk, ME, USA) and incubated for $24 \mathrm{~h}$ under standard culturing conditions. Prior to the assay, SPION suspensions, including $\mathrm{Cu}$-s-SPION10, $\mathrm{Cu}$-s-SPION25, $\mathrm{Ga}$-s-SPION10, and $\mathrm{Ga}$ s-SPION25, were sterilized by filtration with a hydrophilic regenerated cellulose $0.22 \mu \mathrm{m}$ filter. The dose volume of the nanoparticles was kept $<24 \%$ of the total volume. A sample of the filtered particles in cell media at a desired concentration was processed for AAS to confirm the concentration of SPIONs applied to cells. The filtered particles were diluted in complete culture medium and added to triplicate wells spanning a 3-log range of final concentrations (250, $125,62.5,31.2,15.6,7.81$, and $3.91 \mathrm{ppm}$, unless otherwise stated). The cells were then incubated for 24 or $72 \mathrm{~h}$ before $1.0 \mathrm{mM}$ thiazolyl blue tetrazolium bromide (MTT; L11939; Alfa Aesar, Heysham, UK) was added and further incubated for $4 \mathrm{~h}$. The media was removed, and $200 \mu \mathrm{L}$ of dimethyl sulfoxide (DMSO; Sigma-Aldrich) was added to each well. The absorbance at $600 \mathrm{~nm}$ was then measured for the plate in a Victor $^{3} \mathrm{~V}$ microplate reader (1420; Perkin Elmer). $\mathrm{IC}_{50}$ 
values were determined as the drug concentration that reduced the absorbance to $50 \%$ of that in untreated control wells.

\section{Results and discussion}

\section{Quantitative measurements of $\mathrm{Co}^{2+}$} concentration on SPIONs

\section{Radiolabeling of SPIONs with $\left[{ }^{57} \mathrm{Co} /{ }^{n a t} \mathrm{Co}\right]^{2+}$ : effect of $\mathrm{pH}$ and temperature}

Initially we investigated suitable and optimum conditions for radiolabeling. The first parameter we investigated was $\mathrm{pH}$. Inorganic bases are often used to adjust the $\mathrm{pH}$ for radiolabeling reactions by many research groups. ${ }^{26,27}$ Then, $0.1 \mathrm{M} \mathrm{NaOH}$ was first used to adjust the $\mathrm{pH}$ of the reaction mixture to 8.0. However, the use of $\mathrm{NaOH}$ resulted in the $\mathrm{pH}$ becoming excessively high and causing precipitation of $\mathrm{Co}(\mathrm{OH})_{2}$ in the reaction mixture. As shown in Figure 1A, in the control sample without the presence of SPIONs, an extremely low level of activity ( $10 \%$ of the total) was detected in the supernatant after centrifugation when using $\mathrm{NaOH}$ to adjust the $\mathrm{pH}$, whereas when using $0.1 \mathrm{M}$ sodium acetate directly to obtain $\mathrm{pH} 8, \sim 100 \%$ of the total activity remained in the supernatant after centrifugation.

A
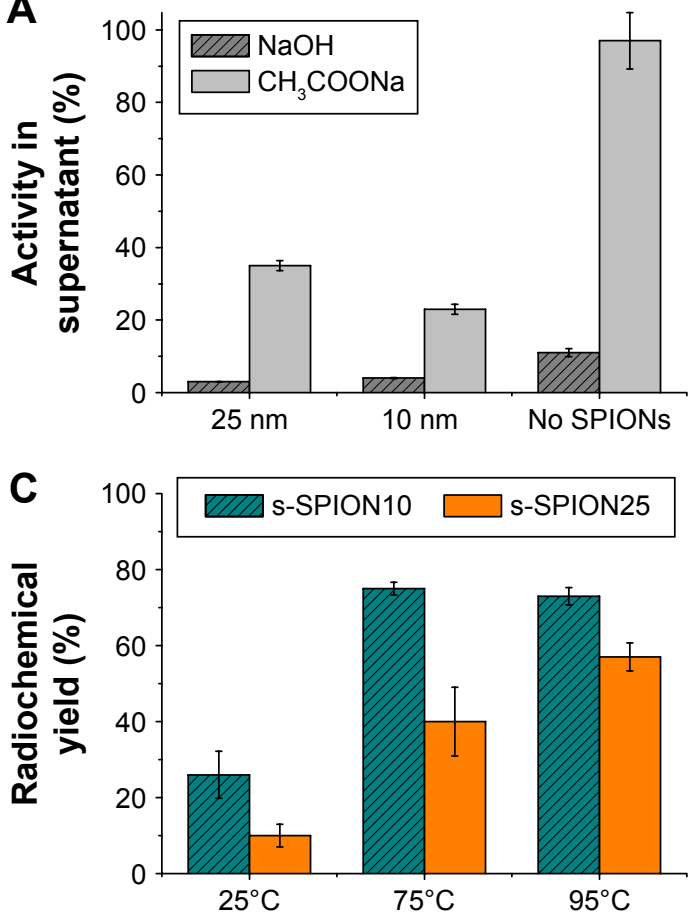

The radiometal labeling was also influenced by the $\mathrm{pH}$ of the reaction mixture. When using sodium acetate buffer, increasing $\mathrm{pH}$ (from 4.0 to $~ 8.0$ ), at the same reaction temperature of $95^{\circ} \mathrm{C}$, resulted in a marked improvement in radiochemical yield for the radiolabeling of SPIONs with $\left[{ }^{57} \mathrm{Co} /{ }^{\text {nat }} \mathrm{Co}\right]^{2+}$. Experimental data shown in Figure 1B illustrate that under low $\mathrm{pH}$ conditions (acetate buffer, $\mathrm{pH}=4.0-5.5$ ), low radiochemical yields, $<20 \%$, were obtained for s-SPION10. The highest radiolabeling efficiency $(\sim 77 \%)$ was obtained when the reaction was carried out using $0.1 \mathrm{M}$ sodium acetate at $\mathrm{pH} \sim 8$. A range of temperatures were also studied to find out an optimum reaction temperature for the labeling reaction. Results shown in Figure 1C illustrate that under the $\mathrm{pH} 8.0$ acetate buffer as the optimum buffer, increasing temperature also significantly improved radiochemical yield for both s-SPION10 and s-SPION25. At room temperature $\left(25^{\circ} \mathrm{C}\right)$, the radiochemical yield with $\left.\left[{ }^{57} \mathrm{Co} /{ }^{\text {nat }} \mathrm{Co}\right]\right]^{2+}$ only reached $26 \%$ and $10 \%$ for s-SPION10 and s-SPION25, respectively. When heating the reaction mixtures to $75^{\circ} \mathrm{C}$ for $30 \mathrm{~min}$, the radiochemical yield was $\sim 75 \%$ and $40 \%$ yield for s-SPION10 and s-SPION 25 , respectively. The highest radiochemical yields, $\sim 75 \%$ and $57 \%$ for s-SPION10 and s-SPION25, respectively, were
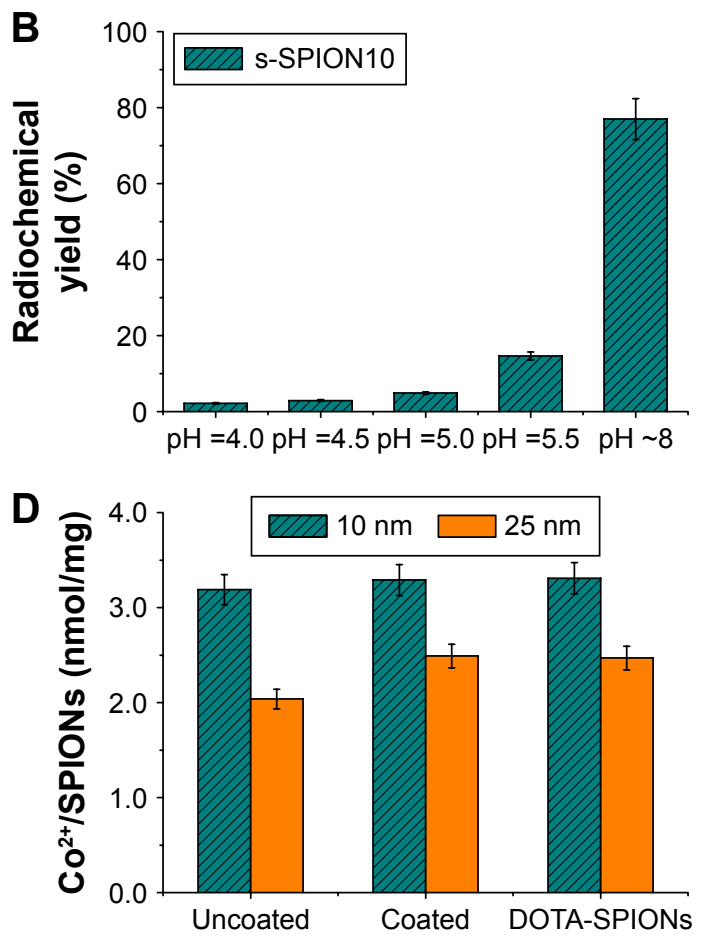

Figure I Radiolabeling SPIONs with radioactive cobalt.

Notes: Dispersion of SPIONs (I wt\%, $50 \mu \mathrm{L}$, s-SPIONI0 or s-SPION25) was added into a solution of $\left[{ }^{57} \mathrm{Co}^{\text {nat }} \mathrm{Co}^{2+}\left(\sim 10 \mathrm{KBq},\left[{ }^{\text {nat }} \mathrm{Co}{ }^{2+}\right]=2 \mathrm{nmol}\right)\right.$ in $(\mathbf{A}) 0 . \mathrm{I} \mathrm{M}$ sodium acetate $(\mathrm{pH} \sim 8)$ or water with $\mathrm{pH}$ adjusted to 8 using $0.1 \mathrm{M} \mathrm{NaOH}$, heated at $95^{\circ} \mathrm{C}$ for $30 \mathrm{~min}$; (B) $0.1 \mathrm{M}$ acetate buffer at different $\mathrm{pH}(4-8)$, heated at $95^{\circ} \mathrm{C}$ for 30 min; (C) $0.1 \mathrm{M}$ sodium acetate $(\mathrm{pH} \sim 8)$, heated at different temperatures of 25,75 , or $95^{\circ} \mathrm{C}$ for $30 \mathrm{~min}$; and (D) $0.1 \mathrm{M}$ sodium acetate ( $\mathrm{pH} \sim 8$ ), heated to $95^{\circ} \mathrm{C}$ for 30 min. Error bars $=$ standard errors, $n=3$.

Abbreviations: nat, natural; DOTA-SPIONs, s-SPIONs with DOTA $\left(1,4,7,10\right.$-tetraazacyclododecane-I,4,7,10-tetraacetic acid) as chelating ligand conjugated to the $\mathrm{NH}_{2}$ end group of the stabilizer; SPIONs, superparamagnetic iron oxide nanoparticles; s-SPIONs, stabilized SPIONS; s-SPIONIO, s-SPIONs with average core diameter of I0 nm; s-SPION25, s-SPIONs with average core diameter of $25 \mathrm{~nm}$. 
obtained when the reaction mixtures were heated at $95^{\circ} \mathrm{C}$ for $30 \mathrm{~min}$. As the same amount of iron oxide mass was used in all cases, the number of particles, ie, the number of reaction sites, for the smaller size particles was significantly higher than that for the bigger ones. This resulted in the higher radiochemical yield for s-SPION10 under the same conditions. From these results, a temperature of $95^{\circ} \mathrm{C}$ and $0.1 \mathrm{M}$ sodium acetate, $\mathrm{pH} 8.0$, were chosen for the radiolabeling of SPIONs with $\left[{ }^{57} \mathrm{Co} /{ }^{\text {nat }} \mathrm{Co}\right]^{2+}$ for further studies.

As the SPIONs were coated with polymer blocks that could potentially chelate to metal ions, it is important to determine whether the $\left[{ }^{57} \mathrm{Co} /{ }^{\text {nat }} \mathrm{Co}\right]^{2+}$ ions bind to the nanoparticle surface or the polymer-coating layers. The uncoated SPIONs, the coated SPIONs as well as the coated SPIONs with 1,4,7,10-tetraazacyclododecane-1,4,7, 10 -tetraacetic acid as chelating ligand conjugated to the $\mathrm{NH}_{2}$ end group of the stabilizer (DOTA-SPIONs) were labeled with $\left[{ }^{57} \mathrm{Co} /{ }^{\text {nat }} \mathrm{Co}\right]^{2+}$ using the same conditions; $0.1 \mathrm{M}$ sodium acetate, at $95^{\circ} \mathrm{C}$ for $30 \mathrm{~min}$. The radioactivity obtained on the nanoparticles (Figure 1D) was similar between three particle types, -3.3 and $2.5 \mathrm{nmol}$ of $\mathrm{Co}^{2+} / \mathrm{mg}$ of 10 and $25 \mathrm{~nm}$ SPIONs, respectively. This result strongly indicates that $\mathrm{Co}^{2+}$ ions are likely deposited on the surface of the iron oxide core, even in the presence of additional chelating ligands.

\section{Loading capacity of $\left[{ }^{57} \mathrm{Co} /{ }^{n a t} \mathrm{Co}\right]^{2+}$ on s-SPIONs}

To further investigate the metal binding capacity of $\mathrm{Co}^{2+}$ on the s-SPIONs, fixed amounts of s-SPIONs $(0.5 \mathrm{mg})$ were added into reaction mixtures with increasing ratios of ${ }^{\text {nat }} \mathrm{Co}^{2+} / 57 \mathrm{Co}^{2+}$
(Figure 2). To minimize the use of higher radioactivity level, we did not aim to extend the duration of a radiolabeling reaction. Thus, we chose a reaction time of $30 \mathrm{~min}$ and evaluated the optimum concentration and conditions to achieve a highest possible radiolabeling yield. The fixed amount of ${ }^{57} \mathrm{Co}^{2+}$ would act as a radiotracer for the experiment. Once the loading limit of nat $\mathrm{Co}^{2+}$ loading was reached, increasing levels of ${ }^{57} \mathrm{Co}^{2+}$ would be found in the supernatant. The reaction conditions were maintained constant; $95^{\circ} \mathrm{C}$ for $30 \mathrm{~min}$; the amount of $\left[{ }^{57} \mathrm{Co} /{ }^{\text {nat }} \mathrm{Co}\right]^{2+}$ led to an elevated level of $\left[{ }^{57} \mathrm{Co} /{ }^{\text {nat }} \mathrm{Co}\right]^{2+}$ in the product (Figure 2A). As expected, the radiochemical yield decreased with increasing ratios of ${ }^{\text {nat }} \mathrm{Co}^{2+}$ to ${ }^{57} \mathrm{Co}^{2+}$ (Figure $2 \mathrm{~B}$ ). Using $400 \mathrm{nmol}$ of ${ }^{n a t} \mathrm{Co}^{2+} / \mathrm{mg}$ of s-SPIONs gave $\sim 170 \mathrm{nmol}$ (yield 42\%) and $110 \mathrm{nmol}$ (yield $28 \%$ ) $\left[{ }^{57} \mathrm{Co} /{ }^{\text {nat }} \mathrm{Co}\right]^{2+} / \mathrm{mg}$ of s-SPION10 and s-SPION25, respectively. Meanwhile, the radiochemical yields reached $90 \%$ for s-SPION10 and $71 \%$ for $\mathrm{s}$-SPION25 within $30 \mathrm{~min}$ of reaction time when only $2 \mathrm{nmol}$ of $\left[{ }^{57} \mathrm{Co} /{ }^{\text {nat }} \mathrm{Co}\right]^{2+} / \mathrm{mg}$ of s-SPIONs were used.

\section{Stability of $\left[{ }^{57} \mathrm{Co} /{ }^{\text {nat }} \mathrm{Co}\right]^{2}$ labeled-SPIONs $\left(\left[{ }^{57} \mathrm{Co} /{ }^{\text {nat }} \mathrm{Co}\right]^{2+}\right.$-s-SPIONs) in simulated}

\section{physiological conditions}

As anticipated, the experimental data showed that the radiolabeled nonstabilized SPIONs were not dispersible at all in aqueous phases. The particles coagulated and/or aggregated and settled down to the bottom of the vial rapidly. Any aggregation or flocculation of particles under physiological conditions would cause the blocking of blood vessels or local retention of particles upon treatment and therefore result in an acute toxicity of the particles.
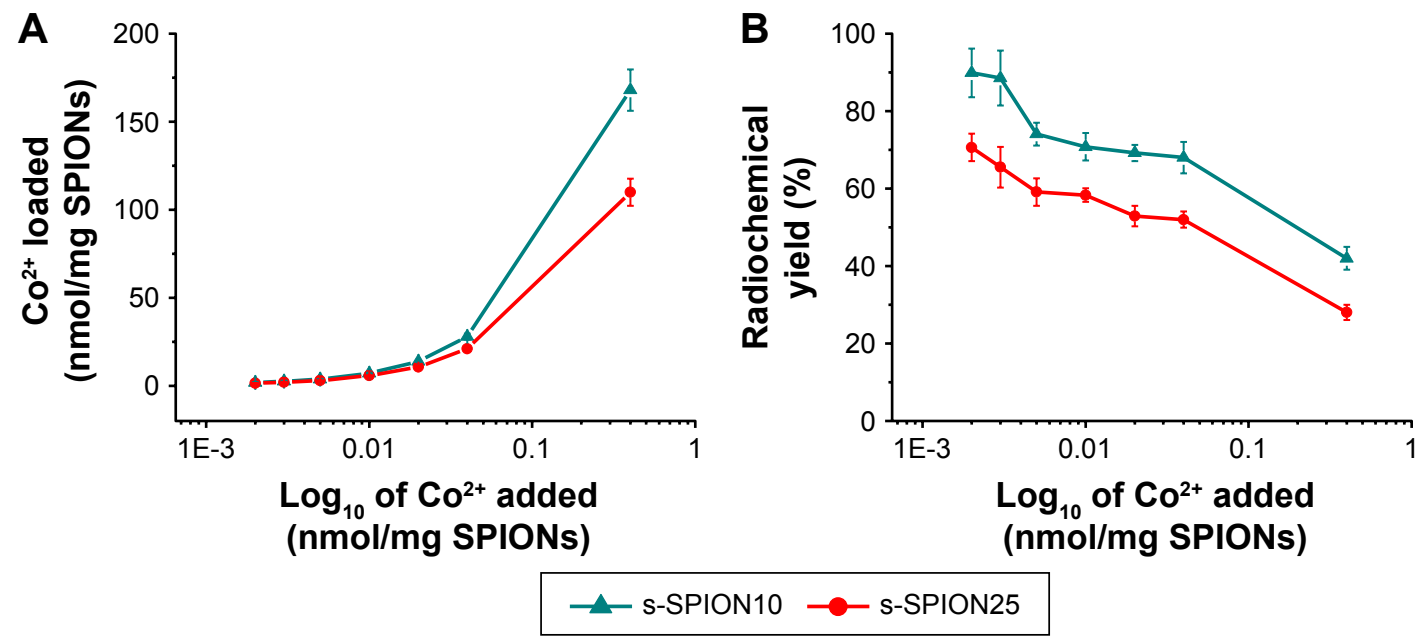

Figure 2 Loading capacity of $\mathrm{Co}^{2+}$ on s-SPIONs after the reaction for $30 \mathrm{~min}$ at $95^{\circ} \mathrm{C}$ in $0.1 \mathrm{M}$ sodium acetate.

Notes: (A) amounts of $\mathrm{Co}^{2+}$ loaded on s-SPIONs (nmol/mg of dry s-SPIONs) and (B) labeling yield (\%) when increasing the amounts of ${ }^{57} \mathrm{Co} / \mathrm{Co}^{2+}$ added in the reaction mixtures. Error bars $=$ standard errors, $n=3$.

Abbreviations: SPIONs, superparamagnetic iron oxide nanoparticles; s-SPIONs, stabilized SPIONs; s-SPIONI0, s-SPIONs with average core diameter of I0 nm; s-SPION25, s-SPIONs with average core diameter of $25 \mathrm{~nm}$. 
On the other hand, due to the coating layer of steric stabilizers, radiolabeling the s-SPIONs did not alter the aqueous-phase dispersability of the initial s-SPIONs. During the radiolabeling, the s-SPIONs remained well dispersed under the harsh reaction conditions, including high concentration of buffer and high temperature (Figure 3A). Radiolabeled s-SPIONs could be easily resuspended and were stable in various aqueous dispersing media, including water, $0.9 \%$ saline, and PBS. The AAS results for the filtered s-SPIONs and labeled s-SPIONs (data not shown) showed that the concentration of iron given by SPIONs did not change after being filtered through a sterile $0.22 \mu \mathrm{m}$ filter (to sterile SPIONs for in vitro cytotoxicity tests), which is another solid evidence of the well dispersed and stable s-SPIONs.

Retention of the radionuclide with the vector is a critical aspect of radiotracer development. Loss of the radionuclide may result in localization in untargeted tissue or untimely excretion, thus it is important that the radionuclides remain bound to the particles. We investigated this property of our particles by incubation of $\left[{ }^{57} \mathrm{Co} /{ }^{n a t} \mathrm{Co}\right]^{2+}$-s-SPIONs in water, saline, and PBS for $24 \mathrm{~h}$. We observed that $<2 \%$ of the total activity was leached out from the cores during this time period, indicating very stable binding of the radiometal (Figure 3B).

\section{Labeling s-SPIONs with PET/SPECT agents}

With the optimization of radiolabeling conditions and the excellent stability of ${ }^{57} \mathrm{Co}$-s-SPIONs, we investigated the radioisotopes potentially useful as SPECT and PET imaging agents, ${ }^{67} \mathrm{Ga}$ and ${ }^{64} \mathrm{Cu}$, respectively.

\section{s-SPIONs labeled with ${ }^{67} \mathrm{Ga}$}

Similar conditions were applied to investigate the reaction between s-SPIONs and ${ }^{67} \mathrm{Ga}$. In this study, a mixture of $\left[{ }^{67} \mathrm{Ga} /{ }^{\text {nat }} \mathrm{Ga}\right]^{3+}$ was prepared by adding $10 \mu \mathrm{L}$ of ${ }^{67} \mathrm{Ga}$ $(\sim 2.5 \mathrm{MBq}$, chloride form in $0.1 \mathrm{M} \mathrm{HCl})$ to $200 \mu \mathrm{L}$ of $0.1 \mathrm{mM} \mathrm{Ga}$ (III) nitrate solution in $0.1 \mathrm{M} \mathrm{HCl}$. The reaction was carried out at either $\mathrm{pH} 5.5(0.1 \mathrm{M}$ acetate buffer) or pH 8 ( $0.1 \mathrm{M}$ sodium acetate) and heated at $95^{\circ} \mathrm{C}$ for $30 \mathrm{~min}$. The radiochemical yield was found to be almost quantitative $(100 \%)$ at both pHs and for both s-SPION10 and s-SPION25 (Figure 4A).

\section{Studying the reactions of s-SPIONs with nonradioactive $\mathrm{Cu}^{2+}$ and $\mathrm{Ga}^{3+}$ and the stability of the products}

The high labeling efficiency of s-SPIONs with ${ }^{57} \mathrm{Co}$ and ${ }^{67} \mathrm{Ga}$ and the stability of the products have been demonstrated. These studies were repeated using nonradioactive cations, including $\mathrm{Cu}^{2+}$ and $\mathrm{Ga}^{3+}$. Furthermore, the colloidal properties of the s-SPIONs, including particle size and stability, and the toxicity of the labeled s-SPIONs were also studied. The amount of metal cations was quantified by GF-AAS. The reactions with $\mathrm{Cu}^{2+}$ or $\mathrm{Ga}^{3+}$ at different $\mathrm{pHs}$ and temperatures were only performed with s-SPION25 (Figure 4B and C). The control reactions, in which no s-SPION25 was added, were carried out simultaneously to monitor for the precipitation of metal oxide or hydroxide in the higher $\mathrm{pH}$ buffers. Under all studied conditions, $100 \%$ of added metal cations were found to remain in the supernatant after centrifugation (data not shown). Similar to what we observed in radioactive labeling
A

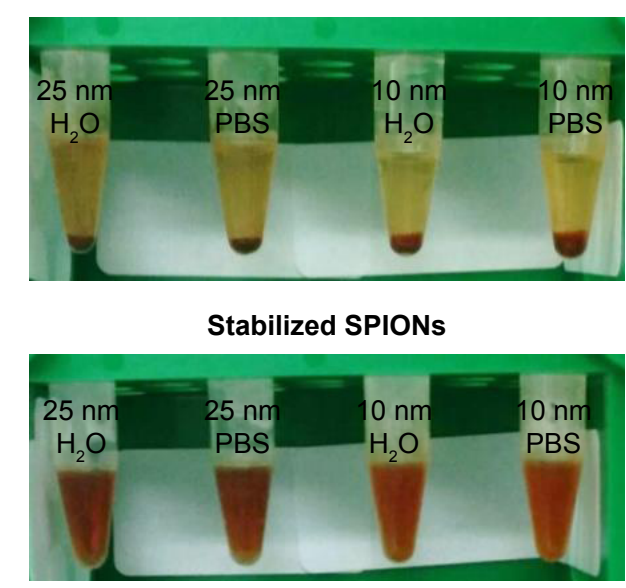

B

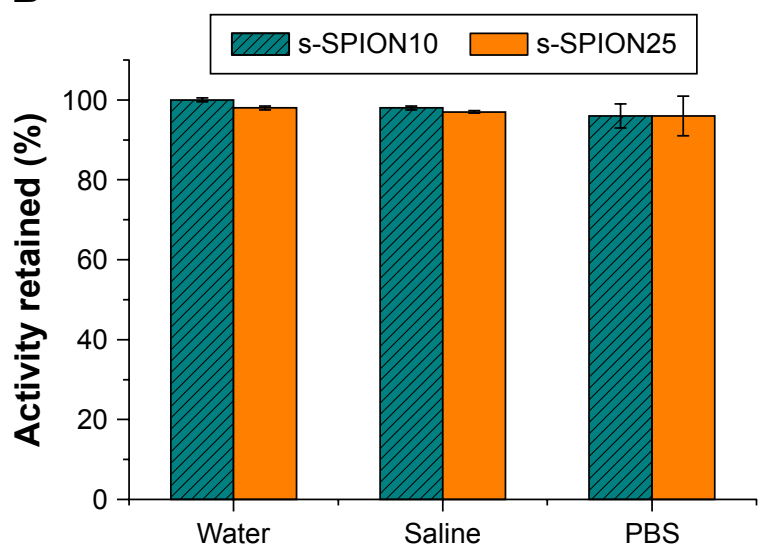

Figure 3 Colloidal and radiochemical stability of SPIONs.

Notes: (A) Well dispersion of the stabilized SPIONs in water and PBS after labeling with ${ }^{57} \mathrm{Co}$ (bottom) in contrast to the unstabilized SPIONs (top) and (B) the activity of ${ }^{57} \mathrm{Co}$ remained on $\left.{ }^{57} \mathrm{Co} /{ }^{n o t} \mathrm{Co}\right]^{2+}$-s-SPIONs after $24 \mathrm{~h}$ under physiological conditions. Error bars = standard errors, $\mathrm{n}=3$.

Abbreviations: nat, natural; SPIONs, superparamagnetic iron oxide nanoparticles; s-SPIONs, stabilized SPIONs; s-SPIONIO, s-SPIONs with average core diameter of $10 \mathrm{~nm}$; s-SPION25, s-SPIONs with average core diameter of $25 \mathrm{~nm}$; NP, nanoparticle; PBS, phosphate-buffered saline. 
A

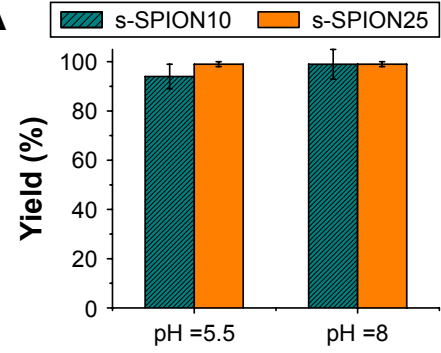

D

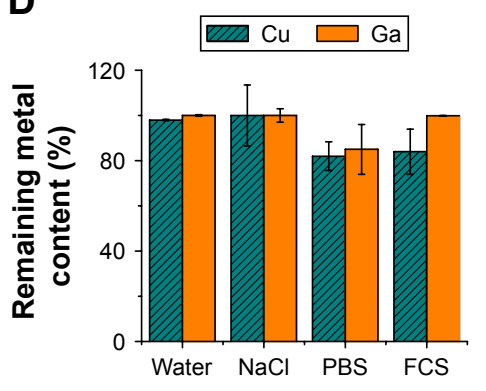

B

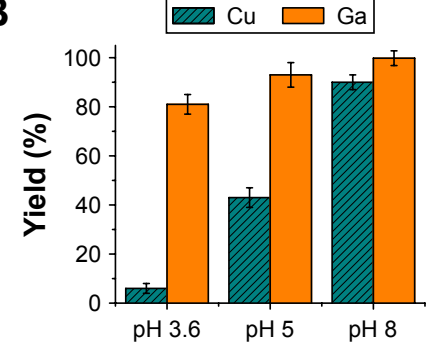

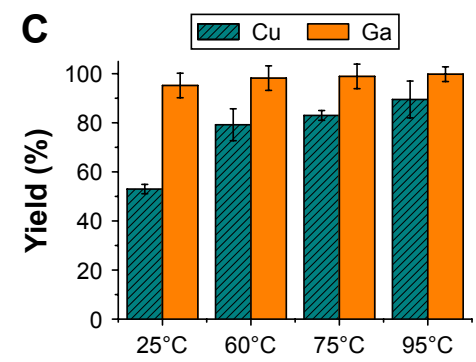

E

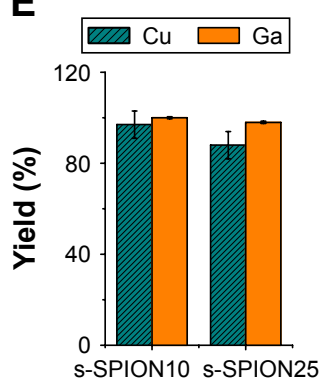

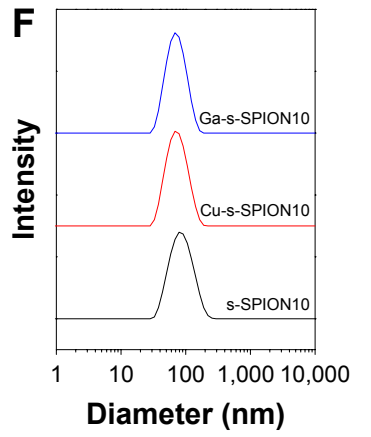

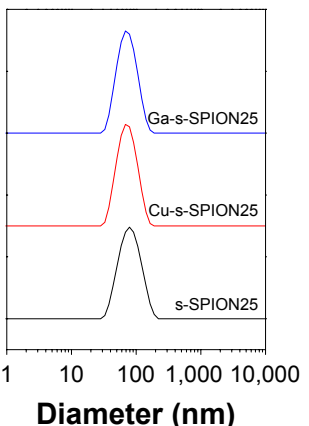

G
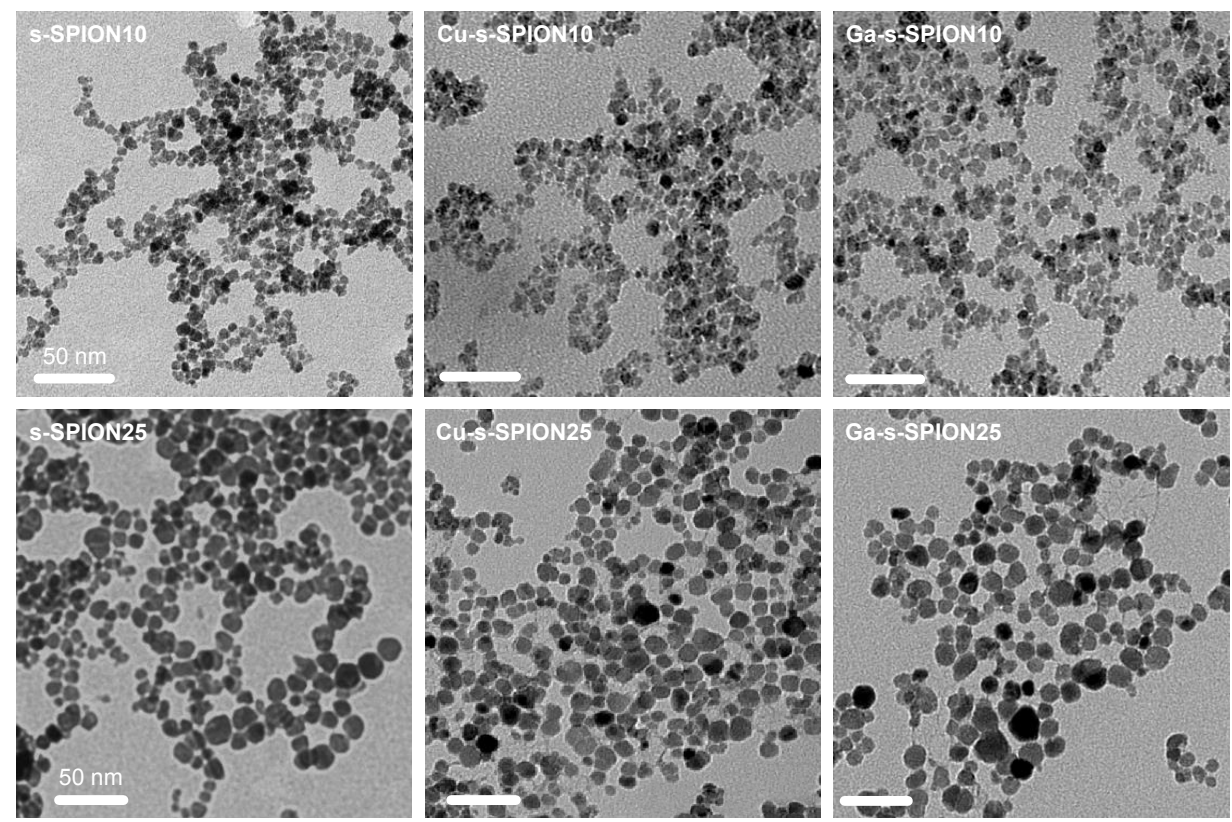

Figure 4 Labeling s-SPIONs with $\mathrm{Ga}$ and/or $\mathrm{Cu}$.

Notes: (A) Labeling efficiency of s-SPIONs (I wt\%, $50 \mu \mathrm{L}$, s-SPIONI0 or s-SPION25) with [ $\left.{ }^{67} \mathrm{Ga} /{ }^{\text {nat }} \mathrm{Ga}\right]^{3+}\left(\sim 125 \mathrm{KBq}, \mathrm{I}\right.$ nmol Ga $\left.{ }^{3+}\right)$ at pH $5.5(0 . \mathrm{M}$ Macetate buffer) or at pH 8 (0.I M sodium acetate), heating at $95^{\circ} \mathrm{C}$ for 30 min. (B and C) Labeling efficiency of s-SPION25 with $\mathrm{Cu}^{2+}$ and $\mathrm{Ga}^{3+}$ (in $0.1 \mathrm{M}$ sodium acetate at different pHs, heating at $95^{\circ} \mathrm{C}$ for $30 \mathrm{~min}$, and at different temperatures, $\mathrm{pH}$ 8, for $30 \mathrm{~min}$ ). Constant amounts of $0.5 \mathrm{mg} s-S P I O N s$ and I nmol of metal ions were used in all reactions. (D) Stability of Cu-s-SPION25 and Ga-s-SPION25 after $24 \mathrm{~h}$ incubation in different media represented by the percentages of $\mathrm{Cu}$ or $\mathrm{Ga}$ remained bound to nanoparticles. (E) Co-labeling of s-SPIONs with $\mathrm{Cu}^{2+}$ and $\mathrm{Ga}^{2+}$ at $95^{\circ} \mathrm{C}$ after 30 min resulted in $\sim 100 \%$ yield for both metal cations. (F) DLS of s-SPIONs before and after labeling with $\mathrm{Ga}^{3+}$ or $\mathrm{Cu}^{2+}$ did not show any changes in the hydrodynamic size. (G) Transmission electron micrographs of the sterically stabilized particles before labeling (s-SPIONIO and s-SPION25) and after labeling with $\mathrm{Cu}^{2+}$ (Cu-s-SPIONIO and Cu-s-SPION25) or with Ga ${ }^{3+}$ (Ga-s-SPIONIO and Ga-s-SPION25); scale bars: $50 \mathrm{~nm}$. Error bars = standard errors, $\mathrm{n}=3$.

Abbreviations: Cu-s-SPIONIO, s-SPIONs with average core diameter of $10 \mathrm{~nm}$ and labeled with Cu; Ga-s-SPIONIO, s-SPIONs with average core diameter of $10 \mathrm{~nm}$ and labeled with Ga; Cu-s-SPION25, s-SPIONs with average core diameter of $25 \mathrm{~nm}$ and labeled with Cu; Ga-s-SPION25, s-SPIONs with average core diameter of $25 \mathrm{~nm}$ and labeled with Ga; SPIONs, superparamagnetic iron oxide nanoparticles; s-SPIONs, stabilized SPIONs; s-SPIONIO, s-SPIONs with average core diameter of $10 \mathrm{~nm}$; s-SPION25, s-SPIONs with average core diameter of $25 \mathrm{~nm}$; PBS, phosphate-buffered saline; FCS, fetal calf serum; DLS, dynamic light scattering.

with ${ }^{67} \mathrm{Ga}$, the reaction of $\mathrm{Ga}^{3+}$ with s-SPION25 was less $\mathrm{pH}$ and temperature dependent. The reaction yields after heating to $95^{\circ} \mathrm{C}$ at $\mathrm{pH} 5$ and 8 were $\sim 93 \%$ and $\sim 99 \%$, respectively (Figure 4B). The yield obtained at $\mathrm{pH} 3.6$ was slightly lower ( $>80 \%)$. Using $0.1 \mathrm{M}$ sodium acetate $(\mathrm{pH} \sim 8), \sim 100 \%$ of
$\mathrm{Ga}^{3+}$ added in the reaction mixture bound to s-SPION25 not only at $95^{\circ} \mathrm{C}$ but also at $25^{\circ} \mathrm{C}, 60^{\circ} \mathrm{C}$, and $75^{\circ} \mathrm{C}$ (Figure $4 \mathrm{C}$ ). In contrast, the reaction of s-SPION 25 with $\mathrm{Cu}^{2+}$ was strongly $\mathrm{pH}$ and temperature dependent as shown in Figure 4B and C. The labeling process required high temperature and high $\mathrm{pH}$ 
in order to obtain a high yield of the product. Specifically, in $0.1 \mathrm{M}$ sodium acetate and heating at $95^{\circ} \mathrm{C}$ for $30 \mathrm{~min}, \sim 90 \%$ of added $\mathrm{Cu}^{2+}$ was deposited on the surface of s-SPION25.

The $C u$-s-SPION25 and $G a$-s-SPION25 were incubated for $24 \mathrm{~h}$ in various media including water, $0.9 \%$ saline, $\mathrm{PBS}$, and $10 \%$ FCS to study the dissociation of $\mathrm{Cu}$ and $\mathrm{Ga}$ from SPIONs into the media. All samples remained dispersed in these media. GF-AAS analyses of $\mathrm{Cu}$ and $\mathrm{Ga}$ concentrations in the supernatant after centrifugation showed that $<1 \%$ of both $\mathrm{Cu}$ and $\mathrm{Ga}$ were released from SPIONs in either water or saline (Figure 4D). Incubation of labeled s-SPIONs in PBS or FCS resulted in $<10 \%$ of $\mathrm{Ga}$ and $\mathrm{Cu}$ leaching. However, this could be due to the presence of s-SPIONs still remaining in the supernatant after centrifugation as the errors were significant.

In addition, using this method, labeling s-SPIONs with both $\mathrm{Cu}^{2+}$ and $\mathrm{Ga}^{3+}$ for hybrid PET-SPECT imaging was successfully carried out. The multiradioisotope-labeled s-SPIONs offer high potential as all-in-one PET/SPECT-MRI agents. Applying reaction conditions that gave the highest efficacy for both $\mathrm{Cu}^{2+}$ and $\mathrm{Ga}^{3+}, 0.1 \mathrm{M}$ sodium acetate and heating at $95^{\circ} \mathrm{C}$ for $30 \mathrm{~min}$, using equal amounts of $\mathrm{Cu}^{2+}$ and $\mathrm{Ga}^{3+}(1 \mathrm{nmol})$ for the reaction with either s-SPION10 or s-SPION25 $(0.5 \mathrm{mg}), \sim 100 \%$ yield and $>90 \%$ yield were achieved for $\mathrm{Ga}^{3+}$ and $\mathrm{Cu}^{2+}$, respectively (Figure 4E).

The hydrodynamic size of the labeled s-SPIONs did not change after going through a tough preparation process involving high salt concentration, high temperature, and highspeed centrifugation (Figure 4F). The TEM images of the SPIONs before and after metal labeling (Figure 4G) showed that the labeling processes did not change the core size or the appearance of the particles significantly. The observed excellent stability properties of these s-SPIONs are crucial for nanomedicine applications as they should ensure their stability during preparation and future investigations in vivo.

It is worth noting that all (radio-)metal-labeling processes described above were robust and reproducible with very small standard errors (Figures 1-4).

\section{Toxicity of the metal-labeled s-SPIONs in vitro}

The toxicity of the s-SPION10 and s-SPION25 in various cell lines was described in our previous reports. ${ }^{10,11,24}$ In order to study the safety of the labeled s-SPIONs by MTT assay, four samples of $\mathrm{Cu}$-s-SPION10, $\mathrm{Cu}$-s-SPION25, $\mathrm{Ga}$-sSPION10, and $\mathrm{Ga}$-s-SPION25 were prepared in larger scale using $10 \mathrm{mg}$ of s-SPION10 or s-SPION25 and $0.6 \mu \mathrm{mol}$ of $\mathrm{Cu}^{2+}$ or $\mathrm{Ga}^{3+}$. The reactions were carried out in $0.1 \mathrm{M}$ acetate buffer $(\mathrm{pH} 8.0)$ at $95^{\circ} \mathrm{C}$ for $30 \mathrm{~min}$, and then the labeled
Table I Concentrations of labeled s-SPIONs used in MTT assay for cell viability

\begin{tabular}{lll}
\hline Sample & {$[\mathrm{Fe}](\mathrm{mM})$} & {$[\mathrm{Cu}]$ or $[\mathrm{Ga}](\mu \mathrm{M})$} \\
\hline Cu-s-SPIONI0 & 50 & 273 \\
Ga-s-SPION10 & 50 & 282 \\
Cu-s-SPION25 & 50 & 234 \\
Ga-s-SPION25 & 50 & 241 \\
\hline
\end{tabular}

Abbreviations: [Cu], copper concentration; [Fe], iron concentration; [Ga], Ga concentration; MTT, thiazolyl blue tetrazolium bromide; Cu-s-SPIONIO, s-SPIONs with average core diameter of $10 \mathrm{~nm}$ and labeled with Cu; Ga-s-SPIONIO, s-SPIONs with average core diameter of $10 \mathrm{~nm}$ and labeled with Ga; Cu-s-SPION25, s-SPIONs with average core diameter of $25 \mathrm{~nm}$ and labeled with Cu; Ga-s-SPION25, s-SPIONs with average core diameter of $25 \mathrm{~nm}$ and labeled with Ga; s-SPIONs, stabilized superparamagnetic iron oxide nanoparticles.

products were collected by centrifugation and washed three times with water prior to redispersing in $1 \mathrm{~mL}$ of water. The concentrations of $\mathrm{Fe}$ and $\mathrm{Cu}$ or $\mathrm{Ga}$ in the final products were quantified by GF-AAS. For the MTT assay, all four samples were diluted with saline to the final concentrations of $50 \mathrm{mM} \mathrm{Fe}$, and concentrations of $\mathrm{Cu}$ and $\mathrm{Ga}$ are given in Table 1. The highest concentration of the labeled s-SPIONs tested on two cell lines was $3.0 \mathrm{mM}$ of Fe (corresponding to $250 \mu \mathrm{g} / \mathrm{mL} \mathrm{Fe}_{2} \mathrm{O}_{3}$ ). No cytotoxicity was observed on L6 muscle cells and 3T3-L1 preadipocytes at the highest concentration tested as shown in Figure 5.

The good dispersity, stability in aqueous media, and noncytotoxicity of the sterically stabilized SPIONs, both before ${ }^{10}$ and after labeling with radiometal and metal ions (as shown in this study), are essential requirements for imaging contrast agents in particular and for any further biomedical application in general.

\section{Conclusion}

SPIONs with excellent MRI-negative contrast were successfully radiolabeled with the SPECT radioisotope, ${ }^{67} \mathrm{Ga}$, or with the PET radioisotope, ${ }^{64} \mathrm{Cu}$, in acetate buffer. The radiochemical yields of radiolabeling with ${ }^{57} \mathrm{Co}$ were found to be strongly dependent on temperature and time for both raw ferro-fluid (unstabilized iron oxide cores) and sterically stabilized SPIONs. No detectable loss of radionuclides from the particles to the aqueous phase in various physiological media was observed. The sterically stabilized radiolabeled s-SPIONs were fully redispersed and remained stable in aqueous buffer solutions such as PBS (Figure 5) and were also shown to be noncytotoxic in vitro. These are crucial properties of nanoparticles for an injectable formulation in biomedical applications.

In addition to the synthesis of the well-defined PET/SPECTMRI, our capability to synthesize SPIONs with heterogeneous coating, which consists of different end functional groups such as $-\mathrm{NH}_{2}$ on the stabilizing molecules, ${ }^{11,24}$ also 

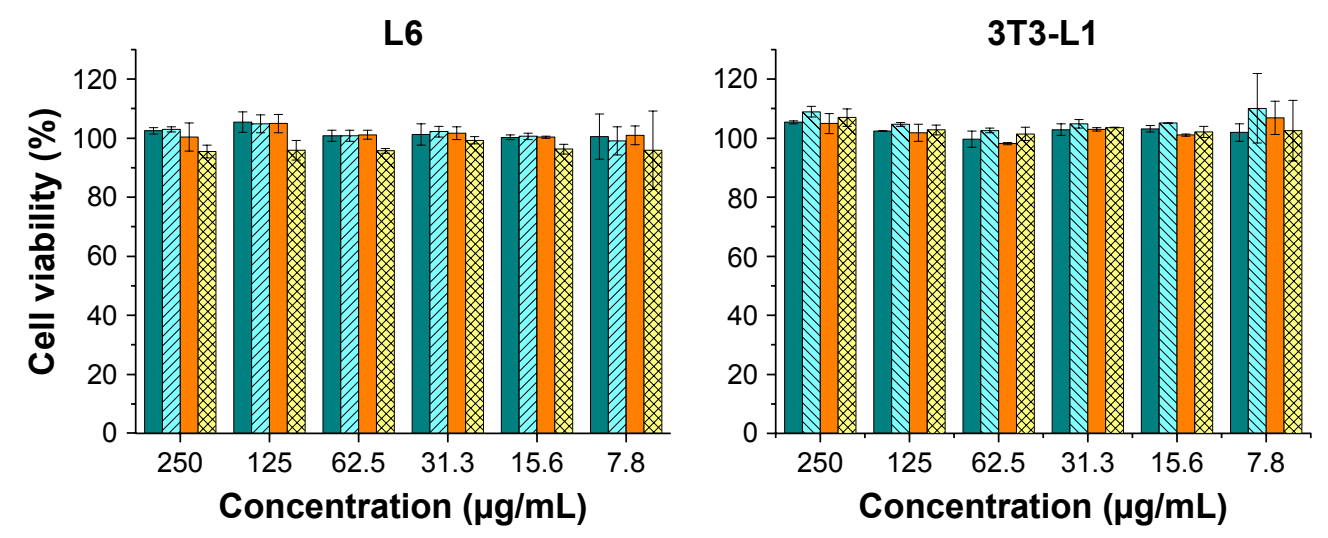

Cu-s-SPION10 VIIIA Cu-s-SPION25

Ga-s-SPION10 Ga-s-SPION25

Figure 5 In vitro cytotoxicity tests for the metal-labeled SPIONs.

Notes: L6 (left) or 3T3-LI (right) cell lines were treated with Cu/Ga-labeled s-SPIONs at the concentration of up to $250 \mu \mathrm{g} / \mathrm{mL}$ for $72 \mathrm{~h}$. No significant toxicity was observed for all samples. Error bars = standard errors, $n=3$.

Abbreviations: SPIONs, superparamagnetic iron oxide nanoparticles; s-SPIONs, stabilized SPIONs; s-SPIONI0, s-SPIONs with average core diameter of I0 nm; s-SPION25, s-SPIONs with average core diameter of $25 \mathrm{~nm}$.

enable us to carry out further postmodification with finely controlled amounts of an appropriate targeting agent and/or fluorescent imaging agent.

This study offers a robust and simple production for a potential next generation of nontoxic multimodality PET/ SPECT-MRI single probe imaging agents.

\section{Acknowledgments}

The authors acknowledge the financial support from Sirtex Medical Ltd. and from AINSE grant (award number ALNGRA15522); and Dr Algi Serelis of DuluxGroup for RAFT reagents. The authors acknowledge the facilities and the technical assistance of the Australian Science and Technology Organisation (ANSTO), and of the Australian Microscopy \& Microanalysis Research Facility (AMMRF) at the Australian Centre for Microscopy and Microanalysis (ACMM), The University of Sydney.

\section{Disclosure}

The authors report no conflicts of interest in this work.

\section{References}

1. Koo H, Huh MS, Sun IC, et al. In vivo targeted delivery of nanoparticles for theranosis. Acc Chem Res. 2011;44(10):1018-1028.

2. Kircher MF, Mahmood U, King RS, Weissleder R, Josephson L. A multimodal nanoparticle for preoperative magnetic resonance imaging and intraoperative optical brain tumor delineation. Cancer Res. 2003; 63(23):8122-8125.

3. Cormode DP, Skajaa T, van Schooneveld MM, et al. Nanocrystal core high-density lipoproteins: a multimodality contrast agent platform. Nano Lett. 2008;8(11):3715-3723.

4. Lin J-J, Chen J-S, Huang S-J, et al. Folic acid-pluronic F127 magnetic nanoparticle clusters for combined targeting, diagnosis, and therapy applications. Biomaterials. 2009;30(28):5114-5124.
5. Ke J-H, Lin J-J, Carey JR, Chen J-S, Chen C-Y, Wang L-F. A specific tumor-targeting magnetofluorescent nanoprobe for dual-modality molecular imaging. Biomaterials. 2010;31(7):1707-1715.

6. Pittet MJ, Swirski FK, Reynolds F, Josephson L, Weissleder R. Labeling of immune cells for in vivo imaging using magnetofluorescent nanoparticles. Nat Protoc. 2006;1(1):73-79.

7. Devaraj NK, Keliher EJ, Thurber GM, Nahrendorf M, Weissleder R. $18 \mathrm{~F}$ labeled nanoparticles for in vivo PET-CT imaging. Bioconjug Chem. 2009;20(2):397-401.

8. Song H-T, Jordan EK, Lewis BK, et al. Rat model of metastatic breast cancer monitored by MRI at 3 Tesla and bioluminescence imaging with histological correlation. J Transl Med. 2009;7:88.

9. Lohrke J, Frenzel T, Endrikat J, et al. 25 years of contrast-enhanced MRI: developments, current challenges and future perspectives. Adv Ther. 2016;33(1):1-28.

10. Pham BTT, Jain N, Kuchel PW, et al. The interaction of sterically stabilized magnetic nanoparticles with fresh human red blood cells. Int J Nanomedicine. 2015;10:6645-6655.

11. Eamegdool SS, Weible MW 2nd, Pham BTT, Hawkett BS, Grieve SM, Chan-ling T. Ultrasmall superparamagnetic iron oxide nanoparticle prelabelling of human neural precursor cells. Biomaterials. 2014; 35(21):5549-5564

12. Torres Martin de Rosales R, Tavaré R, Glaria A, Varma G, Protti A, Blower PJ. 99mTc-bisphosphonate-iron oxide nanoparticle conjugates for dual-modality biomedical imaging. Bioconjug Chem. 2011;22(3): 455-465.

13. Hahn MA, Singh AK, Sharma P, Brown SC, Moudgil BM. Nanoparticles as contrast agents for in-vivo bioimaging: current status and future perspectives. Anal Bioanal Chem. 2011;399(1):3-27.

14. Wehrl HF, Judenhofer MS, Wiehr S, Pichler BJ. Pre-clinical PET/MR: technological advances and new perspectives in biomedical research. Eur J Nucl Med Mol Imaging. 2009;36(suppl 1):S56-S68.

15. Wadas TJ, Wong EH, Weisman GR, Anderson CJ. Coordinating radiometals of copper, gallium, indium, yttrium, and zirconium for PET and SPECT imaging of disease. Chem Rev. 2010;110(5):2858-2902.

16. Werner RA, Bluemel C, Allen-Auerbach MS, Higuchi T, Herrmann K. (68)Gallium- and (90)Yttrium-/(177)Lutetium: "theranostic twins" for diagnosis and treatment of NETs. Ann Nucl Med. 2015;29:1-7.

17. Stelter L, Pinkernelle JG, Michel R, et al. Modification of aminosilanized superparamagnetic nanoparticles: feasibility of multimodal detection using 3T MRI, small animal PET, and fluorescence imaging. Mol Imaging Biol. 2010;12(1):25-34. 
18. Thorek DLJ, Ulmert D, Diop N-FM, et al. Non-invasive mapping of deep-tissue lymph nodes in live animals using a multimodal PET/MRI nanoparticle. Nat Commun. 2014;5:3097.

19. Kiessling F, Mertens ME, Grimm J, Lammers T. Nanoparticles for imaging: top or flop? Radiology. 2014;273(1):10-28.

20. ErranteY, Cirimele V, MallioCA, DiLazzaro V,ZobelBB, QuattrocchiCC. Progressive increase of T1 signal intensity of the dentate nucleus on unenhanced magnetic resonance images is associated with cumulative doses of intravenously administered gadodiamide in patients with normal renal function, suggesting dechelation. Invest Radiol. 2014;49(10): 685-690.

21. Kanda T, Ishii K, Kawaguchi H, Kitajima K, Takenaka D. High signal intensity in the dentate nucleus and globus pallidus on unenhanced T1-weighted MR images: relationship with increasing cumulative dose of a gadolinium-based contrast material. Radiology. 2014;270(3): 834-841.

22. FDA Drug Safety Communication: FDA strengthens warnings and changes prescribing instructions to decrease the risk of serious allergic reactions with anemia drug Feraheme (ferumoxytol). Published March 30, 2015. US Department of Health and Human Services; US Food and Drug Administration. Available from: http://www.fda.gov/ Drugs/DrugSafety/ucm440138.htm. Accessed January 11, 2017.
23. Stendahl JC, Sinusas AJ. Nanoparticles for cardiovascular imaging and therapeutic delivery, part 1: compositions and features. J Nucl Med.2015; 56(10):1469-1475.

24. Bryce NS, Pham BTT, Fong NWS, et al. The composition and endgroup functionality of sterically stabilized nanoparticles enhances the effectiveness of co-administered cytotoxins. Biomater Sci. 2013;1(12): 1260-1272.

25. Pham BTT, Such CH, Hawkett BS. Synthesis of polymeric Janus nanoparticles and their application in surfactant-free emulsion polymerizations. Polym Chem. 2015;6(3):426-435.

26. Normandin MD, Yuan H, Wilks MQ, et al. Heat-induced radiolabeling of nanoparticles for monocyte tracking by PET. Angew Chem Int Ed Engl. 2015;54(44):13002-13006.

27. Boros E, Bowen AM, Josephson L, Vasdev N, Holland JP. Chelatefree metal ion binding and heat-induced radiolabeling of iron oxide nanoparticles. Chem Sci. 2015;6(1):225-236.

28. Ščasnár V, Lier JE. The use of SEP-PAK Sl cartridges for the preparation of gallium chloride from the citrate solution. Eur J Nucl Med Mol Imaging. 1993;20(3):1.
International Journal of Nanomedicine

\section{Publish your work in this journal}

The International Journal of Nanomedicine is an international, peerreviewed journal focusing on the application of nanotechnology in diagnostics, therapeutics, and drug delivery systems throughout the biomedical field. This journal is indexed on PubMed Central, MedLine, CAS, SciSearch $®$, Current Contents $\AA /$ Clinical Medicine,

\section{Dovepress}

Journal Citation Reports/Science Edition, EMBase, Scopus and the Elsevier Bibliographic databases. The manuscript management system is completely online and includes a very quick and fair peer-review system, which is all easy to use. Visit http://www.dovepress.com/ testimonials.php to read real quotes from published authors. 